\title{
DETERMINAÇÃO DOS CUSTOS DE MUDANÇA PARA USUÁRIOS DE TRANSPORTE AÉREO EM RELAÇÃO AO PREÇO DAS TARIFAS
}

\section{Determination of switching costs for air transport users in relation to the price of fares}

Willker Wallisson F. Vieira ${ }^{1}$, Fernando Odilon M. Torres ${ }^{2}$, Marcos André A. de Mendonça ${ }^{3}$, Willer Luciano Carvalho ${ }^{4}$

Recebido em 05 de setembro de 2016; recebido para revisão em 06 de fevereiro de 2017; aceito em 24 de março de 2017; disponível on-line em 19 de abril de 2017.

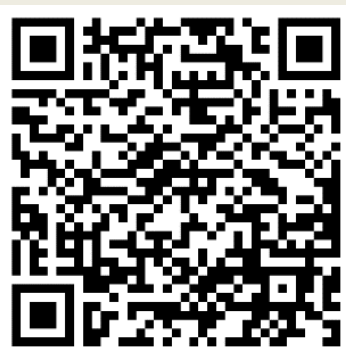

PALAVRAS CHAVE:

Planejamento de transporte;

Transporte aéreo;

Escolha discreta;

Logit multinominal;

Custo de mudança.

\section{KEYWORDS:}

Transportation planning;

Air transport;

Discrete choice; Multinomial logit; Switching costs.

\footnotetext{
* Contato com os autores:

${ }^{1}$ e-mail: willker_w@hotmail.com (W.W. F. Vieira )

Eng. Civil, Escola de Engenharia Civil e Ambiental - EECA, Engenharia Civil, UFG.

2e-mail: engfomt@hotmail.com (F. O. M. Torres )

Eng. Civil, Escola de Engenharia Civil e Ambiental - EECA, Engenharia Civil, UFG.

${ }^{3}$ e-mail: marcosandreamm@gmail.com (M. A. A. Mendonça)

Eng. Civil, Escola de Engenharia Civil e Ambiental - EECA, Engenharia Civil, UFG.

${ }^{4}$ e-mail: willer_carvalho@ufg.br (W. L. Carvalho )

Doutor, Professor, Faculdade de Ciências e Tecnologia - FCT, Engenharia de Transportes, UFG.
}

ABSTRACT: This paper analyzes the degree of loyalty of air transport users of Goiannia's (iscrete choice model logit multinomial, came to the critical percentage change in the price of airline tickets that makes a customer loyal to a company moving to the other services. The results obtained for the strategies, from a better understanding of the airline industry user behavior in Brazil. 


\section{INTRODUÇÃO}

A aviação civil no Brasil sofreu inúmeras transformações desde 1927, ano do voo inaugural da primeira empresa brasileira de aviação civil. De um mercado rudimentar, na década de 20 , em que a constituição de empresas aéreas era livre e a regulação praticamente não existia, o país obteve, ao longo do tempo, um setor com empresas de porte e com marco regulatório definido. Inclusive, a partir de 2005, passou a contar com uma agência reguladora dedicada, a Agência Nacional de Aviação Civil - ANAC (Mckinsey e Company, 2010).

Diante do enorme crescimento deste mercado e do significativo conjunto de companhias aéreas disponíveis, faz-se necessário estudar o seu mercado consumidor e a relação deste com os prestadores de serviço. Uma vez que se deseja o aumento da qualidade e da regulação do serviço oferecido, é imprescindível que se estude o que é preferência, os fatores que levam a uma determinada preferência e, no caso deste trabalho, fazer o levantamento e identificação dos custos que - consumidor deve arcar quando muda de prestador do serviço.

Pode-se afirmar que a preferência por certos produtos ou serviços, em detrimento dos seus concorrentes, reflete o comportamento diante da variedade de opções disponíveis. O caráter básico dos modelos de escolha discreta determina que a probabilidade de uma pessoa fazer certa escolha está diretamente ligada com seus atributos socioeconômicos e da maior vantagem da opção em questão, quando comparada com as demais (Ortuzar e Willumsen, 1994). Dentre os modelos de escolha discreta, destaca-se o Modelo Logit Multinomial (MNL), caracterizado por proporcionar resultados satisfatórios e uma manipulação computacional de fácil acesso.

Para uma melhor compreensão do problema, é preciso introduzir o conceito de Custo de Mudança. Assim, os custos de mudança ou switching costs, na literatura internacional, são custos econômicos e psicológicos que um consumidor pode enfrentar ao trocar de fornecedor ou de empresa prestadora de serviços (Klemperer,
1987). Os Custos de Mudança são assumidos pelo comprador ao mesmo tempo, ou antes, que o substituto consiga fornecer seus benefícios (Porter, 1999; Dikolli; Kinnei; Sedarole, 2007).

Portanto, este trabalho tem o objetivo de determinar qual o custo de mudança para os usuários de transporte aéreo em Goiânia, no que se refere às opções de uso entre as companhias aéreas disponíveis. É de extrema importância que se quantifique tal custo, haja vista que nenhum estudo avançado foi feito nesse sentido no Aeroporto Santa Genoveva, único da capital goiana.

O trabalho estimou o percentual que a passagem de determinada empresa pôde aumentar e que, mesmo assim, o usuário prosseguiu fiel a esta companhia aérea. Para isso, o modelo de escolha discreta utilizado foi o logit multinomial e a coleta de dados foi realizada por preferência declarada. Dessa forma, os resultados contribuem para um melhor entendimento do mercado do transporte aéreo em Goiânia, possibilitando às empresas do setor atuarem no mercado buscando agregar clientes a suas empresas, e traçar estratégias de mercado.

\section{MODELAGEM DE ESCOLHA DISCRETA}

Segundo Souza (2002) as teorias de escolha discreta têm como objetivo transformar as hipóteses sobre os anseios do tomador de decisão em uma função de demanda que expresse a ação de um tomador de decisão sob certas situações. Diversas teorias que procuram explicar o comportamento do tomador de decisão podem ser encontradas em diversas literaturas. Ben-Akiva e Lerman (1985) descrevem as teorias com maior importância.

Souza (2002) afirma que a teoria de escolha probabilística de utilidade randômica é a mais utilizada e recomendada para o desenvolvimento de modelos de preferência declarada. De acordo com Ortuzar e Willumsen (1994), a teoria de utilidade randômica é a base teórica ou o paradigma para a geração de modelos de escolha discreta.

Segundo Souza (2002), a probabilidade de 
uma alternativa i ser escolhida é igual à probabilidade que a utilidade desta alternativa $i$ $\left(U_{\text {in }}\right)$ seja igual ou maior que as utilidades das demais alternativas que estão presentes no grupo de escolha, de acordo com a Equação 1:

$$
P(i / C n)=P(U i n>U j n, j \in C n) \quad \text { Eq. [1] }
$$

\subsection{FUNÇÃO DE UTILIDADE}

De acordo com a teoria de escolha probabilística de utilidade randômica, a função de utilidade é representada como sendo a soma do termo constante $\left(V_{i}\right)$ com a perturbação aleatória, apresentada na Equação 2.

$$
\left(\varepsilon_{\mathrm{i}}\right): \mathrm{U}_{\mathrm{in}}=\mathrm{V}_{\mathrm{in}}+\varepsilon_{\mathrm{in}}
$$

Louviere et al (2000) apud Medeiros (2007) defendem uma função do tipo linear para a utilidade. A componente determinística da função é representada pela Equação 3:

$$
V_{\text {in }}=\sum_{k=1}^{K} \beta_{k} X_{\text {ink }}
$$

\section{Em que:}

$\mathrm{V}_{\mathrm{in}}$ : utilidade da alternativa i para um indivíduo $\mathrm{n}$; $\mathrm{X}_{\mathrm{ink}}$ : valor do atributo $\mathrm{K}$ para uma alternativa i para um indivíduo $\mathrm{n}$;

$\beta_{\mathrm{k}}$ : coeficiente do modelo para o atributo $\mathrm{K}$;

K: quantidade de atributos de cada alternativa.

As funções de utilidade utilizadas neste trabalho e que são modeladas pelo software Biogeme (Bielaire, 2001) são do tipo linear in the parameters (linear nos parâmetros).

Segundo Ben-Akiva e Lerman (1985), as funções linear in the parameters são um bom tipo de função para refletir alguma teoria que temos acerta da influência de vários elementos $X$ na função de utilidade e que tem propriedades computacionais que tornam fácil a estimativa de seus parâmetros desconhecidos. Tais funções são dadas da seguinte forma, onde cada alternativa tem sua própria função de utilidade, como apresentado na Equação 4 e 5:

$$
\begin{array}{cr}
V_{\text {in }}=\beta_{1} X_{i n 1}+\beta_{2} X_{i n 2} & +\beta_{3} X_{i n 3}+\cdots+ \\
\beta_{k} X_{i n k} & \text { Eq. [4] } \\
V_{j n}=\beta_{1} X_{j n 1}+\beta_{2} X_{j n 2} \\
\beta_{k} X_{j n k}
\end{array}
$$

Em que:

$V_{\text {in: }}$ Utilidade da alternativa i para um indivíduo n; $\mathrm{X}_{\text {ink }}$ : Valor do atributo K para uma alternativa i para um indivíduo $\mathrm{n}$;

$\beta_{\mathrm{k}}$ : Coeficiente do modelo para o atributo $\mathrm{K}$.

Os primeiros coeficientes $\beta$ são tratados como constantes das utilidades e os demais são os coeficientes dos modelos para os atributos em consideração.

\subsection{MODELO LOGIT MULTINOMIAL (MNL)}

De acordo com Medeiros (2007), a melhor maneira de se representar os erros aleatórios é através da distribuição normal, da qual é originado o modelo Probit. Porém, como esse modelo tem dificuldades devidas aos problemas originados da programação, surgiram os modelos logit multinomial (MNL). Estes modelos proporcionam resultados satisfatórios e uma manipulação computacional amigável. Assim, de acordo com Medeiros (2007) este é o modelo mais difundido em análises de escolha discreta, e este será o modelo utilizado neste trabalho.

A principal hipótese do MNL é a chamada hipótese IID, onde, segundo Medeiros (2007), os erros aleatórios são identicamente distribuídos e independes.

A Propriedade da independência de alternativas irrelevantes IIA, de acordo com Souza (2002), é um dos aspectos mais discutidos do modelo MNL. Tal propriedade sustenta que a taxa das probabilidades de escolha entre duas alternativas, para um indivíduo determinado, não é afetada por qualquer outra alternativa. Esta propriedade é consequência da hipótese IID.

\subsubsection{Probabilidade de escolha}

Considerando que os termos aleatórios são idênticos e a partir de manipulações algébricas, a expressão fechada que define o modelo logit é a 
da Equação 6 (Medeiros, 2007):

$$
P_{n}(i)=\frac{e^{V_{i n}}}{\sum_{j=C(n)} e^{V_{j n}}}
$$

\section{Em que:}

$P_{n}(i)=$ probabilidade de escolha de uma alternativa i por um indivíduo n;

$C(n)=$ conjunto de alternativas $j$ disponíveis para o indivíduo $\mathrm{n}$;

$\mathrm{V}_{\text {in }}=$ utilidade da alternativa i para um indivíduo $\mathrm{n}$; $\mathrm{V}_{\mathrm{jn}}=\mathrm{utilidade}$ da alternativa $\mathrm{j}$ para um indivíduo $\mathrm{n}$.

\subsubsection{Calibração}

De acordo com Medeiros (2007), a calibração de um método de escolha discreta consiste em um método interativo em que são testados vários conjuntos de variáveis e estruturas de modelo com o objetivo de descobrir o modelo que tem a melhor representação da opção dos tomadores de decisão. O método mais utilizado para a calibração dos modelos logit multinomial é baseado no princípio estatístico da máxima verossimilhança. Tal método tem como objetivo, a partir de certa amostra, a estimativa dos parâmetros do modelo maximizando a probabilidade de aquisição do evento particular analisado (Medeiros, 2007). Usa-se a função logverossimilhança para a determinação da máxima verossimilhança em procedimentos numéricos, isso ocorre devido a ela ser mais tratável numericamente.

\subsubsection{Avaliação}

A avaliação de um modelo MNL normalmente é feita baseando-se em dois parâmetros: índice $\rho^{2}$ e teste-t (Medeiros, 2007).

Medeiros (2007), explica que o teste-t faz a avaliação de que a variável em questão contribui ou não para a explicação do modelo. Assim, para mais de 30 observações, se os valores, em módulo, do teste-t serem maiores do que 1,96 significa que a variável em questão tem um efeito significante e deverá ser mantida no modelo.

0 índice $\rho^{2}$, de acordo com Medeiros (2007), faz a avaliação do ajuste geral do modelo e pode variar de 0 (nenhum ajuste) a 1 (perfeito ajuste). Segundo Ortuzar e Willumsen (1994) valores de $\rho^{2}$ que estão próximos a 0,4 são usualmente considerados como excelentes ajustes.

Quando se espera que uma variável contribua positivamente para a sua utilidade, seus coeficientes devem ter sinal positivo e vice-versa. Assim, segundo Medeiros (2007), os sinais dos coeficientes das variáveis são avaliados de modo a se verificar se elas estão de acordo com o esperado.

\subsubsection{Elasticidade}

Sabe-se que no mercado econômico atual, se o preço diminui o consumo aumenta e se o preço aumenta o consumo diminui. Isto é claramente a lei da demanda. Não é diferente no mercado de transportes e para se medir a influência do preço na decisão dos consumidores, pode-se usar a definição de elasticidade. Elasticidade pode ser definida como a mudança percentual, no consumo de um bem ou de um serviço, causada por uma variação de $1 \%$ em seu preço (Medeiros, 2007).

A Equação 7 define a elasticidade do modelo logit multinomial (Medeiros, 2007):

$$
E\left[P_{i}, X_{i k}\right]=\beta_{k} X_{i k}\left(1-P_{i}\right) \quad \text { Eq. [7] }
$$

\section{Em que:}

$\mathrm{E}[\mathrm{Pi}, \mathrm{Xik}]=$ elasticidade da probabilidade de escolha da empresa $\mathrm{i}$ em relação ao atributo Xik, considerando-se o valor Xk para a alternativa i (Xik); $\beta \mathrm{k}=$ coeficiente do atributo $X \mathrm{k}$;

$\mathrm{Pi}=$ probabilidade de escolha da alternativa $\mathrm{i}$.

\section{DADOS DE ESCOLHA DISCRETA: PREFERÊNCIA DECLARADA E PREFERÊNCIA REVELADA}

Segundo Medeiros (2007), todas as partes do processo de modelagem dependem dos tipos de dados que serão coletados. Os principais dados usados na modelagem de escolha discreta são dos tipos: preferência declarada e preferência revelada.

Segundo Medeiros (2007), os dados de preferência revelada se caracterizam por escolhas que já foram feitas pelos consumidores de certo 
serviço ou produto, dentro de um conjunto de opções existentes. Brandli e Reineck (2005) caracterizam os dados de preferência revelada como "o mundo como ele é" e explicam que a utilização da técnica de preferência revelada se baseia nas observações das escolhas reais dos tomadores de decisão. De acordo Adamowicz et al (1994), a limitação da forma de obtenção de dados por preferência revelada se refere à identificação de decisões que os consumidores tomariam em situações nunca vivenciadas antes. Kroes e Sheldon (1988), explicam que a obtenção da variação suficiente de dados PR, para poder se analisar todas as variáveis de interesse, pode ser difícil.

Segundo Green e Srinnasan (1990), os Métodos de Preferência Declarada (MPD) podem ser definidos como conjunto qualquer de métodos decomposicionais cujo objetivo é a estimativa da estrutura de preferências de consumidores, dando a eles todas as avaliações de um conjunto de alternativas pré-estabelecidas em termos de níveis ou atributos diferentes. Brandli e Reineck (2005) caracterizam os dados de Pesquisa Discreta - PD como "o mundo como ele poderia ser" e explicam que o seu princípio básico é a apresentação de um grupo de opções hipotéticas, para o consumidor, das quais ele deverá escolher somente uma.

\subsection{TIPOS DE QUESTIONÁRIOS PD}

Segundo Hensher (1993), as alternativas de questionário PD podem ser apresentadas de três formas diferentes: ranking, rating e choice. Os questionários utilizados neste trabalho são do tipo choice.

Segundo Medeiros (2007), no experimento do tipo de choice (escolha discreta) são apresentadas várias opções ao respondente, e este escolherá a de sua preferência. De acordo com Hensher (1993), a atração pelos experimentos de choice tem como fundamento as grandes incertezas acerca da qualidade dos dados rating e ranking. A vantagem desse experimento está na simplicidade com a qual são apresentados os questionários aos entrevistados, em relação aos outros dois tipos.

\section{SWITCHING COSTS (CUSTOS DE MUDANÇA)}

Os custos de mudança, ou switching costs, na literatura internacional, são custos econômicos e psicológicos que um consumidor pode enfrentar ao trocar de fornecedor ou de empresa prestadora de serviços (Klemperer, 1987). Os Custos de Mudança são assumidos pelo comprador concomitante ou mesmo antes que o substituto possa fornecer seus benefícios (Porter, 1999; Dikolli; Kinnei; Sedarole, 2007). Vários autores listaram e dissertaram sobre os mais variados tipos de Custos de Mudança e seus respectivos impactos na decisão de compra. Identificaram custos subjetivos, de difícil mensuração monetária, e custos econômicos, que interferem diretamente na questão financeira das empresas.

Um importante autor nesse estudo é Klemperer (1987a, 1987b). Segundo ele, os Custos de Mudança podem ser divididos em: custos de transação; custos de aprendizagem; e custos artificiais, chamados também de contratuais. Tais custos são explicados como:

- Custos de Transação: custos que se incorporam por clientes quando da transação econômica, como, por exemplo, no fechamento de uma conta-corrente em um banco e a abertura de outra conta-corrente em um banco concorrente.

- Custos de Aprendizagem: trata-se do investimento do cliente para atingir o mesmo nível de conhecimento e controle com o novo serviço contratado. Ocorrem quando, por exemplo, troca-se de programas de computadores para aumentar a eficiência de determinado serviço, mas, por estes serem mais sofisticados, necessita-se investir no aprendizado do operador do sistema para executar as ferramentas eficazmente. 
- Custos Artificiais: São os custos comumente vistos para rescisão contratual, materializando-se como uma maneira das organizações penalizarem os clientes que desejam finalizar o compromisso contratual. Essas indenizações merecem atenção por parte dos contratantes, pois há a possibilidade de representarem uma parcela considerável dos Custos de Mudança (Shapiro e Varian, 1999). São também classificados como custos artificiais aqueles incorridos a terceiros pela perda das vantagens adquiridas em programas de fidelidade.

No setor de transporte aéreo, os switching costs estão enquadrados nos custos artificiais ao se referirem às perdas de benefícios que sofrem os passageiros quando da troca de companhia aérea, representando uma parcela não negligenciável dos custos incorridos pelos usuários.

Embora o alto investimento, os programas de fidelidade geram a possibilidade das companhias aéreas estabelecerem uma elasticidade dos seus preços (Figer, 2007), já que comprar uma passagem mais barata em empresa concorrente, significa perder oportunidade de acumular milhas na empresa atual. Com isso, há a tendência dos clientes continuarem comprando os serviços de determinada empresa, mesmo o custo da passagem sendo maiores que de outras. É neste contexto que o trabalho se insere: estimar o limite percentual que a passagem de determinada empresa pode ser maior e que, ainda assim, o cliente continue fiel desta. Para tanto, foram coletadas as preferências declaradas dos passageiros do aeroporto de Goiânia em um determinado trecho de voo, a respeito das principais empresas que oferecem o serviço.

\section{ESTUDO DE CASO}

A seguir, serão apresentadas as seções referentes ao estudo de caso no aeroporto Santa Genoveva em Goiânia.

\subsection{CARACTERIZAÇÃO DA LINHA AÉREA OPERACIONAL (GOIÂNIA-GUARULHOS)}

A linha operacional escolhida tem origem no Aeroporto Santa Genoveva, em Goiânia-GO e destino ao Aeroporto Internacional de Guarulhos, em Guarulhos-SP. Como critério de pesquisa, estudou-se a linha operacional com viagens diretas, ou seja, sem qualquer escala em outro aeroporto.

Sabe-se que o voo direto entre Goiânia e Guarulhos dura, aproximadamente, 1 hora e 40 minutos. Inúmeras viagens são realizadas neste sentido. Segundo informações obtidas diretamente com a INFRAERO, as empresas que oferecem estes voos, direto e sem escala, são: TAM, GOL, AZUL e AVIANCA. A GOL oferece 27 voos semanais, a AZUL 18, a AVIANCA 14 e a TAM 12.

\subsection{COLETA E TRATAMENTO DE DADOS}

Como já mencionado em outros tópicos, o processo de coleta de dados foi realizado pelo Método de Preferência Declarada. Foram preparados questionários que colocaram os entrevistados diante de uma situação hipotética na qual, dentre algumas opções dispostas, eles deveriam escolher apenas uma.

Nos questionários, primeiramente se procurou obter informações relevantes para a pesquisa: sexo do entrevistado, fidelização ou não a alguma companhia aérea, motivo da viagem e por quem (empresa, recursos próprios etc.) tal viagem estivesse sendo custeada. Logo após, foi determinada a pergunta chave do questionário: "Imagine que você esteja comprando uma passagem aérea de Goiânia para Guarulhos com seu próprio recurso. Dentre as opções abaixo, qual você realmente escolheria? Escolha somente uma por cartão!". Então, foram dispostos 17 cartões, como o exemplificado a seguir pela Figura 1.

O termo "C1", apresentado na parte inferior do cartão, indica que este é o primeiro dos dezessete cartões de opções do questionário. No primeiro cartão, o preço hipotético da passagem aérea foi o mesmo para as quatro companhias aéreas.

Nos cartões posteriores, este valor foi sendo alterado em uma companhia aérea e mantido 
nas demais. Estas alterações tiveram a alteração de 10 ou 20\%, tanto para mais como para menos. A Tabela 1 representa os valores das tarifas que foram representados em cada cartão de escolha.

Portanto, diante de cada cartão, o entrevistado deveria escolher somente uma alternativa, marcando nas letras (A, B, C e D) da parte superior do cartão e, consequentemente, optando pela companhia aérea com o seu respectivo preço hipotético de passagem.

Foram entrevistadas 235 pessoas. Por motivo de inconsistência de respostas, 46 questionários foram descartados. Logo, 189 questionários (3213 observações) foram utilizados nos resultados do trabalho. Atingiu-se um índice de confiança de $90 \%$ com um erro amostral de $\pm 6 \%$.

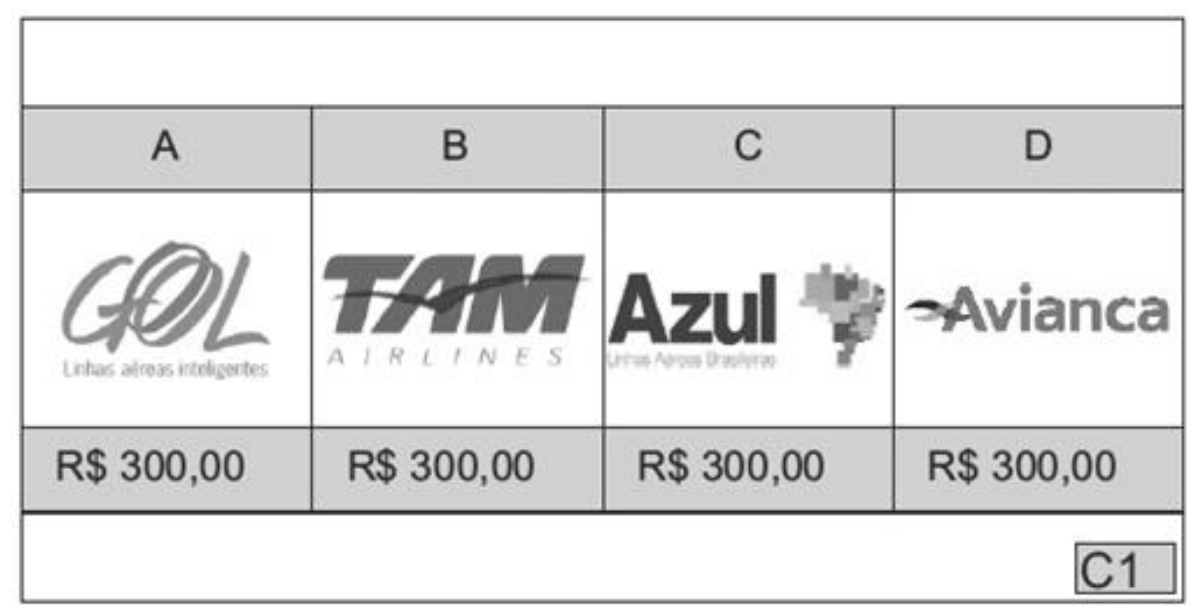

FIGURA 1: Modelo dos cartões de opções apresentados no questionário. FONTE: Autoria própria

TABELA 1: Valores das tarifas em $R \$$ em cada um dos cartões no questionário.

\begin{tabular}{ccccc} 
Cartão & GOL & TAM & AZUL & AVIANCA \\
\hline C1 & 300 & 300 & 300 & 300 \\
C2 & 330 & 300 & 300 & 300 \\
C3 & 300 & 330 & 300 & 300 \\
C4 & 300 & 300 & 330 & 300 \\
C5 & 300 & 300 & 300 & 330 \\
C6 & 360 & 300 & 300 & 300 \\
C7 & 300 & 360 & 300 & 300 \\
C8 & 300 & 300 & 360 & 300 \\
C9 & 300 & 300 & 300 & 360 \\
C10 & 270 & 300 & 300 & 300 \\
C11 & 300 & 270 & 300 & 300 \\
C12 & 300 & 300 & 270 & 300 \\
C13 & 300 & 300 & 300 & 270 \\
C14 & 240 & 300 & 300 & 300 \\
C15 & 300 & 240 & 300 & 300 \\
C16 & 300 & 300 & 240 & 300 \\
C17 & 300 & 300 & 300 & 240 \\
\hline
\end{tabular}

FONTE: Autoria própria 


\subsection{ESTIMATIVA DA FUNÇÃO UTILIDADE}

Para se estimar os parâmetros da função de utilidade, foi utilizado o software Biogeme (Bielaire, 2001) que faz a estimativa para funções linear in the parameters.

As funções para cada uma das empresas são apresentadas nas Equações 8 a 11 respectivamente:

$$
\begin{array}{r}
U_{g o l}=\text { Cgol }+ \text { Custo } * \text { Tarifa } \\
U_{\text {tam }}
\end{array}
$$

Em que:

Tarifa_((nome) $)=$ Valor do atributo preço da passagem para GOL, TAM, AZUL e Avianca;

$U$ (nome) $=$ Utilidades da GOL, TAM, AZUL e Avianca; $C$ (nome $)=$ Constantes da função de utilidade para GOL, TAM, AZUL e Avianca (parâmetros a serem estimados);

Custo $=$ Parâmetro a ser estimado referente ao preço da passagem.

\subsection{RESULTADOS}

Para calcular os custos de mudança utilizou-se a metodologia empregada por Luchessi et al (2013), onde calculou-se a probabilidade agregada variando-se, nas funções de utilidade, o valor do atributo da tarifa TAM e mantendo-se o valor das tarifas das outras empresas em seus valores médios (no caso deste trabalho, o valor médio refere-se ao valor base de $\mathrm{R} \$ 300,00$ ). Segundo Ciarilini (2008), a agregação de probabilidades normalmente é utilizada quando o pesquisador está interessado em alguma medida agregada, como a reação esperada pela mudança de algum fator ou a probabilidade média de uma população. Neste trabalho, a agregação de probabilidades foi estimada pelo método denominado de naive approach. Para calcular as probabilidades agregadas por este método, simplesmente seguiram-se os seguintes passos:

1. Calculou-se a utilidade das empresas GOL, AZUL e Avianca, utilizando os parâmetros estimados pelo software para as equações 9 a 11. Tais utilidades foram calculadas com o valor do atributo tarifa fixado no valor médio $\mathrm{R} \$ 300,00$. Estas utilidades são fixas.

2. Calculou-se a utilidade da empresa TAM com os parâmetros estimados para a equação 8. Tal utilidade foi calculada fazendo-se um incremento no valor do atributo da tarifa TAM em relação ao valor base (TarifaTam $=300,00+$ incremento). Esta utilidade varia (diminui) de acordo com o incremento da tarifa.

3. Utilizando-se a fórmula de probabilidade do modelo logit multinomial (equação 6), calculou-se a probabilidade para cada uma das empresas. Tais probabilidades foram calculadas com a utilidade da empresa TAM variando com a sua tarifa e a utilidade das demais empresas fixadas com o valor da tarifa em sua média. Para cada incremento no valor da tarifa TAM, calcula-se as probabilidades de escolha de cada uma das quatro empresas.

4. Variando-se o valor da tarifa TAM com vários incrementos diferentes, foram feitos os gráficos que demostram as probabilidades de escolha de cada uma das empresas de acordo com o incremento da tarifa TAM.

5. Os custos de mudança são indicados pelas intersecções das linhas (nos gráficos) das probabilidades de escolha das outras empresas com a da TAM.

Além de calcular os custos de mudança para o caso geral, em que se considera toda amostra, também se calculou segregando a amostra em grupos mutuamente exclusivos. Dessa forma, como amostras diferentes geram resultados diferentes, para cada caso, calculou-se a elasticidade da probabilidade de escolha da empresa TAM em relação ao atributo preço da passagem considerando o valor de $\mathrm{R} \$ 300,00$ para a passagem da TAM. O calculo das elasticidades tem a função de se inserir mais um parâmetro para 
podermos fazer comparações entre os grupos. As elasticidades foram calculadas pelo método utilizado por Medeiros (2007), onde:

1. Para se calcular as probabilidades de escolha, se calcula as utilidades Ui, $n$ de cada alternativa, para cada uma das empresas. Como são 17 cartões de escolha e cada empresa possui uma alternativa em cada cartão, cada empresa terá suas 17 utilidades calculadas. Tais utilidades são calculadas utilizando-se as Equações de 1 a 4, com os parâmetros estimados pelo software, e utilizando-se o valor do atributo da tarifa, de cada empresa, que está presente em cada um dos 17 cartões. Após os cálculos dessas utilidades, calculase a probabilidade de escolha da empresa TAM com a Equação 12:

2. Após, calcula-se a elasticidade da probabilidade de escolha da empresa TAM, em relação ao atributo preço da passagem, com a equação 7.

A seguir, serão demostrados os resultados. Os resultados possuem uma tabela com os valores dos testes e parâmetros das funções de utilidade, calculados pelo software, e da elasticidade da probabilidade de escolha da empresa TAM em relação ao atributo preço da passagem considerando o valor de $\mathrm{R} \$ \mathbf{3 0 0 , 0 0}$ para a passagem da TAM.

Para o caso em que foi considerada toda amostra, os parâmetros das funções de utilidade, testes e elasticidade TAM estimados estão representados na Tabela 2 . O gráfico das probabilidades agregadas está representado pela Figura 1.

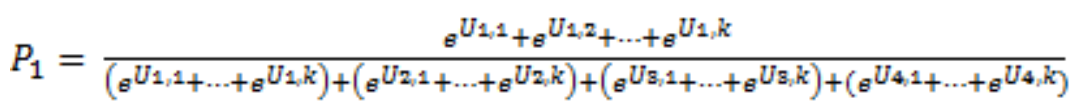

Em que:

P1= probabilidade de escolha da alternativa TAM;

Un,1...Un, $\mathrm{k}=$ utilidades das alternativas $1 . . .17$ das empresas GOL, TAM, AZUL e Avianca. Para

TAM, $n=1$.

TABELA 2: Testes, parâmetros e elasticidade TAM.

\begin{tabular}{lcc}
\multicolumn{2}{c}{ Testes } \\
\hline Teste-t (CUSTO) & $-31,71$ \\
$\rho^{2}$ & 0,24 \\
\hline \multicolumn{3}{c}{ Parâmetros } \\
\hline CUSTO & $-0,0363$ \\
C $_{\text {GOL }}$ & $-0,501$ \\
C $_{\text {TAM }}$ & 0,79 \\
C $_{\text {AZUL }}$ & 0,497 \\
CAvianca & & $-0,786$ \\
\hline
\end{tabular}

Elasticidade TAM

$-6,0018$

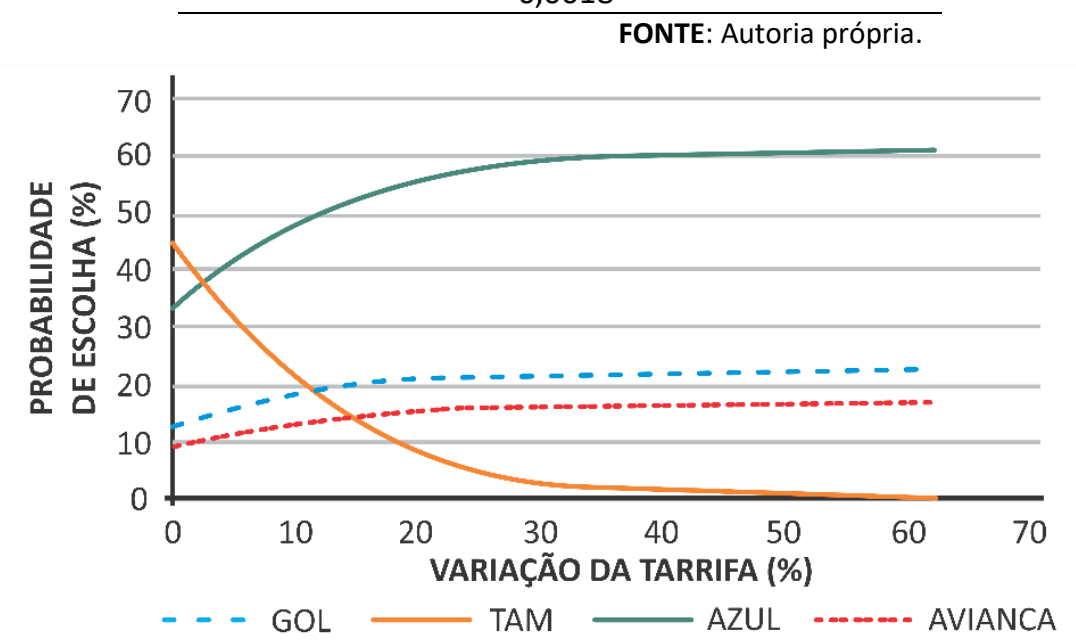

FIGURA 1: Probabilidades de escolha com variação da tarifa TAM.

FONTE: Autoria própria. 
Na Figura 1, pode-se ver que o custo de mudança da empresa TAM para a empresa AZUL refere-se a um incremento de $3 \%$ do valor da tarifa da TAM. O custo de mudança da empresa TAM para a empresa GOL refere-se a um incremento de $12 \%$. A mudança para a empresa Avianca ocorre com um incremento de $15 \%$ na tarifa da TAM. A elasticidade TAM indica que com o aumento de $1 \%$ do valor da sua tarifa a demanda cairia em $6 \%$.

\section{CONCLUSÕES}

A aviação civil é um tema indispensável para o cenário econômico atual. Em um mundo no qual viagens aéreas, curtas ou longas, são cada vez mais necessárias e rotineiras, torna-se necessário o estudo deste tipo de transporte assim como dos fatores que o permeiam.

Logo, analisar preferências e fidelidades do mercado consumidor de transporte aéreo é consequentemente imprescindível. As pesquisas e estudos que foram realizados por este trabalho no Aeroporto Santa Genoveva, Goiânia-GO, foram de certa forma raros. Fez-se eminente conhecer melhor o comportamento e as caraterísticas dos consumidores de um aeroporto que já é o 160 mais rentável do território brasileiro.

Estudos desta modalidade são extremamente interessantes para as companhias aéreas, por se tratar de um mercado bastante competitivo no Brasil. Se analisados e compreendidos da maneira correta, podem tornarse poderosas armas de direcionamento de marketing e consequentemente meios de obtenção de novos clientes fiéis.

Notou-se que o método de pesquisa por Preferência Declarada (MPD) realmente é de fácil aplicação e traz resultados satisfatórios. Igualmente, pôde-se comprovar a eficácia do modelo logit multinomial no que diz respeito principalmente à sua manipulação.

A maneira apresentada de se determinar os custos de mudança através de modelos e dados de preferência declarada têm um enorme potencial de aplicabilidade no mercado. Com mais estudos semelhantes, de modo a se chegar na excelência de aplicabilidade destes métodos, as empresas terão em suas mãos métodos eficazes e relativamente baratos de se analisar e compreender o comportamento dos usuários de transporte aéreo, possibilitando traçar estratégias de mercado para agregar clientes a seus serviços.

\section{REFERÊNCIAS BIBLIOGRÁFICAS}

Adamowicz, W.; Louviere, J.; Williams, M. Combining revealed and stated preference methods for valuing environmental amenities. Journal of Environmental Economics and Management, v. 26, 1994, p. 271-292.

Ben-Akiva, M; Lerman, S. Discrete choice Analysis: Theory and Application to Travel Demand. Cambridge, Cambridge University Press, 1895.

Bielaire, M. BIOGEME: A free package for the estimation of discrete choice models, Switzerland, 3rd Swiss Transportation Research Conference, 2003.

Brandli, L. L.; Reineck, L. F. M. As abordagens dos modelos de preferência declarada e revelada no processo de escolha habitacional. Ambiente Construído, Porto Alegre, 2005 v. 5, n. 2, p.51-75, jun.

Dikolli, S. S.; Kinney Jr, W.; Sedatole, K. L. Measuring Customer Relationship value: The role of switching cost. Contemporary Accounting Research, 2007, v. 24, n. I, p. $93-132$, Spring.

Figer, V. Programas de fidelização e seu impacto no regime de concorrência na indústria aérea americana. Rio de Janeiro, 2007. Dissertação de Mestrado. Programa de Pós Graduação em Economia da PUC-Rio Pontifícia Universidade Católica do Rio de Janeiro (PUC-RJ), 2007.

Green, P.E., Srinnasan, V.. Conjoint analysis in marketing: New developments with implications for research and practice. Journal of Marketing, 1990, Vol. 54, n. 4, Pages 3-19.

Hensher, D. Stated Preference Analysis of Travel choices: The State of Pratice. Tranportation Research, 1994, №21, pg. 107-133.

Klemperer, P. (1987a) The competitiveness of markets with switching costs. Rand Journal of Economics, 1987, v. 18 , n. 1, p. 138-150, Spring.

Klemperer, P. (1987b) Markets with consumer switching costs. Quarterly Journal of Economics, 1987, v. 102, n. 2, p. 375-394.

Kroes, E. P.; Sheldon, R. J. Stated Preference Methods. Journal of Transport Economics and Policy,1998, jan., pg $11-20$.

Louviere, J.J; Hensher, D.A; Swait, J.D. Stated choice 
Methods - Analysis and Application. Cambridge, Cambridge University Press, 2000.

Lucchesi, S.; Nodari, C.; Larranaga, A.; Senna, L. A fidelização dos Clientes às companhias aéreas através de análises de preferência declarada. Anpet, XXVII, 2013.

Medeiros, H. C. Análise das preferências dos usuários do transporte rodoviário de passageiros: estudo de caso na linha Florianópolis (SC) - Porto Alegre (RS). $134 \mathrm{f}$. Dissertação (Mestrado) - Curso de Engenharia de Produção, Departamento de Programa de Pós-graduação em Engenharia de Produção, UFSC, Florianópolis, 2007.

Mckinsey e Company. Estudo do Setor de Transporte Aéreo do Brasil: Relatório Consolidado. BNDES, Rio de Janeiro, 2010, 1 a edição.

Ortuzar, J; Willumsen, L. G. Modeling Transport. John Wilwy e Sons, New York, USA, 1994.

Porter, M. E. How competitive forces shape strategy. Article - Faculty e research - Harvard Business Review, Mar./Abr, 1979.

Shapiro, C.; Varian, H. Information Rules: A Strategic Guide to the Network Economy Strategy/Technology. Harvard Business School Press, 1999.

Souza, C. M. Métodos de Preferência Declarada: Aplicações no setor de transportes aquaviários. $125 \mathrm{f}$. Dissertação (Mestrado) - Programa de Pós Graduação em Engenharia Oceânica, Departamento de Coppejufrj, UFRJ, Rio de Janeiro, 2002. 